\title{
Geographisch-künstlerische Stadtforschung
}

Ein Drei-Schritt-Verfahren zur Erschließung der Vielheit sozialräumlichen Wissens

\author{
Lea Bauer \\ Eva Nöthen
}

\begin{abstract}
Um urbane Problemlagen in ihrer Vielschichtigkeit zu beforschen, bedienen sich Kunst und Wissenschaft jeweils spezifischer Vorgehensweisen. Trotz wiederkehrender gegenseitiger Bezugnahmen sind systematische Überlegungen zu Anschlussstellen an künstlerische Vorgehensweisen im (sozial)wissenschaftlichen Kontext nach wie vor rar. Die Erkenntnispotenziale von Aufarbeitungs- und Artikulationsformen der bildenden und darstellenden Künste werden jedoch zunehmend - beispielsweise in der kritischen Stadtforschung thematisiert. Dabei werden erste Forschungspraxen entwickelt, die künstlerische und wissenschaftliche Vorgehensweisen in transdisziplinären Projektkonstellationen integrieren. Anliegen dieses Textes ist es, anknüpfend an einen Beitrag von Cecilie Sachs Olsen und Sabeth Tödtli zum partizipatorischen Potenzial künstlerischer Stadtforschung, einen theoretisch begründeten methodologischen Ansatz geographisch-künstlerischer Forschungspraxis zu entwickeln, um Stadt aus kritischer Perspektive anders sehen, verstehen und mitgestalten zu können. Dazu werden Henri Lefebvres Theorie zur sozialen Produktion des Raums und Julian Kleins Konzept von künstlerischer Forschung zusammengeführt. Das zentrale Ergebnis dieser Zusammenführung ist ein Drei-Schritt-Verfahren, das (1) die Vielfalt ästhetischer und semiotischer Wahrnehmungen sozialräumlicher Situationen versammelt; (2) deren Beziehungen hinterfragt und spielerisch neu denkt; sowie (3) diese in einer Weise präsentiert, die dazu einlädt, an der Auseinandersetzung mit pluralen, scheinbar inkommensurablen Wahrnehmungs- und Sichtweisen auf Stadt teilzuhaben. Die theoretischen Überlegungen werden am Beispiel ausgewählter Projekte des Architekturkollektivs raumlabor-berlin aus den Jahren 2006 bis 2017 illustriert.
\end{abstract}

Ersteinreichung: 24. September 2019; Veröffentlichung online: 26. November 2021

An English abstract can be found at the end of the document.

\section{Einleitung: Der Vielheit von Raum und Wissen begegnen}

Unter einer stark befahrenen Hochstraße in Duisburg pustet ein Ventilator durchscheinende Kunststofffolie zu einer großen begehbaren Blase auf (vgl. Abb. 1)[1]. Im Innern der Blasekochen und essen Menschen. Fürwenige Stunden wird der unwirtliche Platz unter der Hochstraße zu einem Ort gemeinschaftlicher Zusammenkunft und gastfreundschaftlicher Heiterkeit. Das Küchenmonument des Architekturkollektivs raumlabor-berlin[2] ist eine mobile Skulptur, die - anders als konventionelle Skulpturen im öffentlichen Raum - nur temporär existiert. An unterschiedlichen Plätzen installiert und durch verschiedene Bespielungen inszeniert, schafft sie zeitlich begrenzte Gemeinschaften, gibt Anlass für Austausch und gestaltet die Stadt an Orten, die für die Stadtplanung eine Herausforderung darstellen. Der Rückgriff 


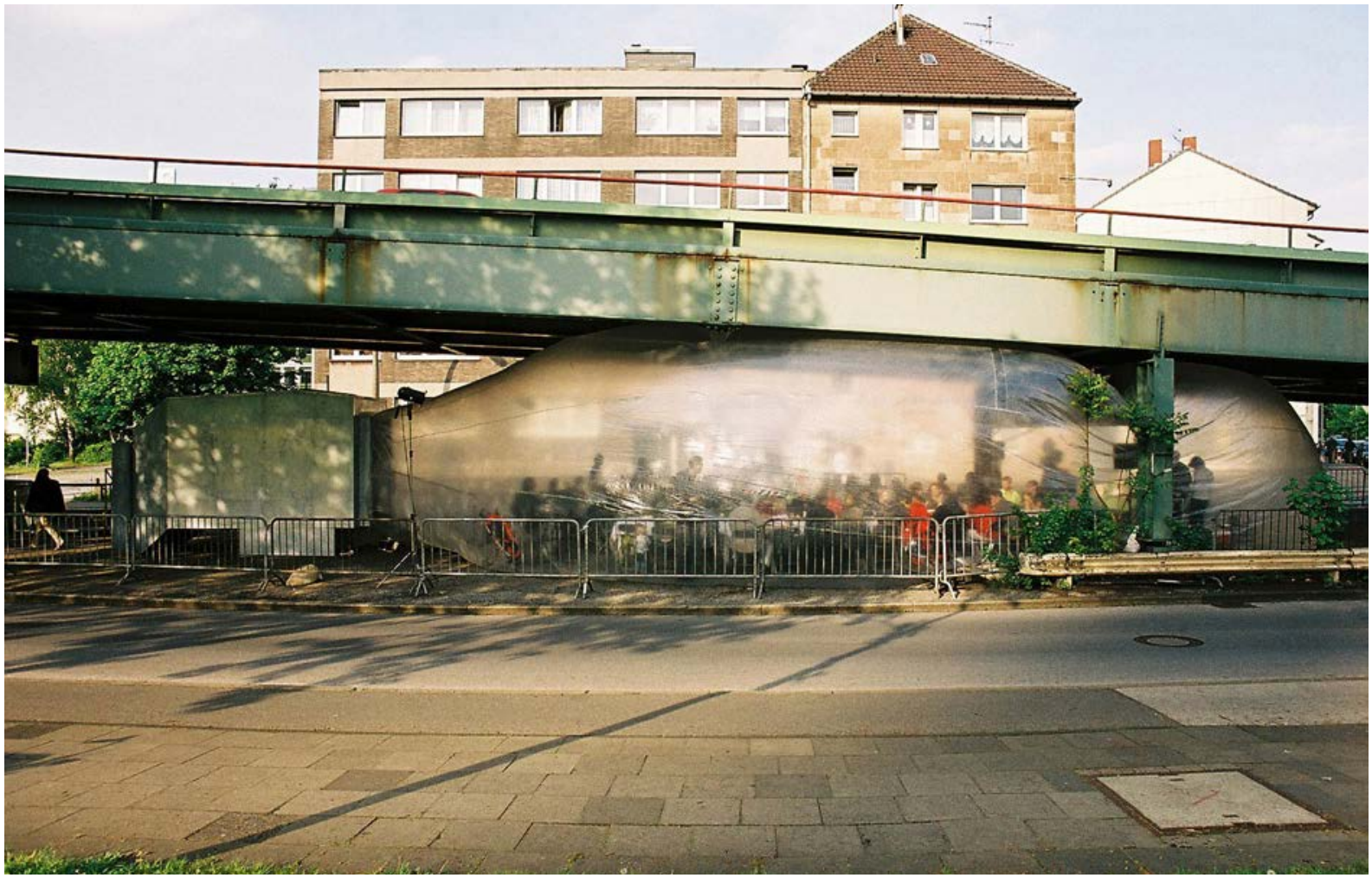

auf unkonventionelle und experimentelle Praktiken des kollektiven SichAuseinandersetzens mit Örtlichkeiten als Beitrag zu einer selbstbestimmten Stadtnutzung ist eine typische Arbeitsweise von raumlabor-berlin (o. J . b). Die beteiligten Architekt_innen zielen darauf ab, mit raumverändernden Eingriffen eine andere Wahrnehmung und Imagination des städtischen öffentlichen Raums anzustoßen. Raumlabor-berlin ist ein Beispiel aus einem Abb. 1 Das Küchenmonument, 2006, raumlabor-berlin (Quelle: Marco Canevacci, raumlaborberlin) Spektruman Initiativen, dieaus einem wissenschaftlich-künstlerisch transdisziplinären Ansatz heraus Perspektiven entwerfen, diedurch Einbezugsinnlichästhetischer und deutenderWahmehmungsweisen den Möglichkeitsraumvon Praktiken der Wissens- und Raumproduktion zum Städtischen[3] erweitern.

Urbane Räume, so sie durch Prozesse der Segregation, Polarisierung, Gentrifizierung oder Privatisierung geprägt sind, sind zunehmend sozialräumlich fragmentiert (vgl. Heeg 2014). In Praktiken wie der oben geschilderten Intervention an der Schnittstelle von Architektur, Stadtplanung und Kunst zeigt sich, dass dieAushandlung von Verantwortlichkeiten längst nicht mehr allein auf der Ebene und in der Sprachevon Politikund Wissenschaft geführt wird. Vielmehr deutet sich in der Praxis des Integrierens von Ausdrucks- und Aufarbeitungsformen der bildenden und darstellenden Künste einerseits sowie wissenschaftlicher Herangehensweisen andererseits an, dass weder der eine noch der andere Ansatz ausreicht, um die Vielschichtigkeit urbaner Problemlagen aufzuspüren, zu analysieren und zu kommunizieren. Anliegen dieses Textes ist es daher, einen theoretisch begründeten methodologischen Ansatz geographisch-künstlerischer Forschungspraxis zu entwickeln.

Künstlerische Forschung ist einer von mehreren Begriffen,[4] mit denen theoretische Perspektiven und praktische Formate bezeichnet werden, die sich mit dem epistemischen Potenzial der Verbindung künstlerischer und wissenschaftlicher Praxen auseinandersetzen. Die Recherche nach und die Sichtung von Beiträgen zu dieser Debatte eröffnen ein Feld, das heterogene theoretische und empirische Bezüge aufweist. Den Ausgangspunkt für die 
folgenden Überlegungen und begrifflichen Entlehnungen bildet ein Beitrag der Geographin Cecilie Sachs Olsen und der Architektin und Urbanistin Sabeth Tödtli - beide auch Gründerinnen des sozial-artistischen Stadtlabors zURBS. In ihrem Aufsatz entwickeln die Autorinnen in Anknüpfung an aktuelle sozial- und raumtheoretische Debatten drei „Thesen zur Vielheit von Raum, von Wissen und dem Potential der Kunstpraxis als kollektive Stadtforschung“" (Sachs Olsen/Tödtli 2016: 187).[5] Die These der Vielheit von Raum versteht Raum als eine niemals fixierte Gegebenheit, die These der Vielheit von Wissen betont die Partialität von Wissen und die These zum Potenzial von Kunstpraxis als kollektiver Stadtforschung versteht sozial engagierte Kunstpraxis als eineVariantekollektiver Stadtforschung. Letztere ist durch ihre spezifischen Methoden besonders geeignet, um „eine Vielheit von Raum und eine Vielheit von Wissen sichtbar zu machen und daraus den Raum für alle möglichen Zukünfte offen zu halten“ (ebd.: 191). Mit der dritten, alle drei Aspekte verbindenden These fassen die Autorinnen somit Kunstpraxis als eine partizipatorische politische Praxis, die eine Vielheit möglicher Raumgestaltungen skizziert. Ausgehend von einer Kritik an „konsensgetriebene[n] urbane[n] Entscheidungsfindungsprozesse[n] “ (ebd.: 189) im Rahmen stadtplanerischer Bürgerbeteiligung, die Wissen als lokal vorliegende Ressource versteht, weisen Sachs Olsen und Tödtli Methoden der Kunstpraxis eine bedeutsame Rolle für eine Etablierung anderer, partizipatorischer Weisen der Stadtgestaltung zu. Die spezifische Kompetenz künstlerischer Methoden sehen sie insbesondere im genauen Hinschauen und der Vorstellungskraft. Dadurch wird es möglich, eine Vielheit lokaler Bezüge von Bewohner_innen und Nutzer_innen als ein immer im Entstehen begriffenes plurales und zugleich partielles Wissen zu verstehen. Eine solche Kunstpraxis kann, so Sachs Olsen und Tödtli, (Re-)Imaginationen schaffen, die „Brüche, Meinungsverschiedenheiten, Antagonismus und Wertepluralismus förder[n]“ (ebd.: 189), indem sie eine Vielheit raumbezogener Wissensweisen in Kopräsenz bringen und so deren Koexistenz denkbar machen (ebd.: 191). Das partizipatorische Potenzial sehen Sachs Olsen und Tödtli darin, dass kollektiv-künstlerische Stadtforschung eine Teilhabe am Um- und Neudenken städtischer Räume ermöglicht und sichtbar macht (ebd.: 202). Zugespitzt formulieren siediesalsForderungnach einem Recht auf eine künstlerische (Re-)Imagination der Stadt (ebd.: 197).

Während der Beitrag von Sachs Olsen und Tödtli vor allem eine theoretische Ausarbeitung des partizipatorischen Potenzials von Kunstpraxis als kollektive Stadtforschung auf Basis umfangreicher Praxiserfahrungen beinhaltet, zielt der vorliegende Text darauf ab, in diesem Sinne ein methodologisches Konzept für eine geographisch-künstlerische Stadtforschung zu entwickeln. Hierfür werden Theorien herangezogen, die zwar nicht Teil der Argumentation von Sachs Olsen und Tödtli sind, sich jedoch geradezu anbieten, um die Thesen einer Vielheit von Raum und einer Vielheit von Wissen als Grundlage einer geographisch-künstlerischen Stadtforschung methodologisch weiterzudenken: Soklärt Kapitel 2 mitdemAnsatzder sozialen Produktion des Raumsnach dem Soziologen und Philosophen Henri Lefebvre (2006 [1974]) in einer aktuellen Lesart die raumtheoretische Verortung des Beitrags und arbeitet diesen als Ansatz zur Beforschung der Vielheit von Raum heraus. Hier werden die drei Dimensionen Repräsentationen des 
Raums, Räume der Repräsentation und Räumliche Praxis rekonstruiert. In Kapitel 3 wird mit dem Ansatz des Komponisten und Theaterregisseurs J ulian Klein (2018) künstlerische Forschungspraxis im Konzept von vier Wahrnehmungsweisen verortet und als artistic mode zur Beforschung der Vielheit von Wissen beziehungsweise der Herausarbeitung heterogener, ästhetischer und semiotischer Wahrnehmungen eingesetzt. Diese Vorüberlegungen zur forschungspraktischen Erschließung der Vielheit von Raum und Vielheit von Wissen aufgreifend erfolgt in Kapitel 4 die Skizzierung des methodologischen Beitrags dieses Textes. Dabei wird geographischkünstlerische Stadtforschung als ein Drei-Schritt-Verfahren vorgestellt und am Beispiel der Projektreihe mobile activators des bereits eingeführten Architekturkollektivs raumlabor-berlin illustriert. Es wird gezeigt, inwiefern künstlerische Forschung eine gewinnbringende Erweiterung einer kritischreflexiven, engagierten, partizipatorischen Stadtforschung sein kann. Abschließend wird in Kapitel 5 das Anliegen des Beitrags zusammengefasst und ein Ausblick auf weiteren Entwicklungsbedarf hinsichtlich konkreter forschungspraktischer Handlungsoptionen gegeben. Schließlich werden forschungsethische Herausforderungen des Ansatzes aufgezeigt.

\section{Die soziale Produktion des Raums als Ansatz zur Beforschung der Vielheit von Raum}

Seit Mitte der 2000er Jahre finden Lefebvres theoretische Überlegungen zur sozialen Produktion des Raums (Lefebvre 2006 [1974]) zunehmend Beachtung und Anwendung in der (kritischen) Stadtforschung. Ausgehend von unterschiedlichen Interpretationen der von Lefebvreentwickelten Raumtriade(n) und deren Übertragungauf dieAnalyseraum-zeitlicher Phänomene und Prozesse in Bezug auf gesellschaftliche Wirklichkeiten finden sich empirische Arbeiten beispielsweise zu Formen der Raumaneignung von Stadtbewohner_innen (Bertuzzo 2009), zu Alltagspraktiken in Stadtquartieren (Vogelpohl 2012), zu gärtnerischen Materialisierungen unter staatlicher Einflussnahme (Exner/Schützenberger 2015) oder zu städtischen Krisenprotesten (Mullis 2017a) im Kontext neoliberaler Stadtentwicklungsprozesse. Im Folgenden wird Lefebvres Ansatz der sozialen Produktion des Raums in Grundzügen rekonstruiert und als binäres Modell zur Analyse von Konflikten im sozialen Raum interpretiert. Damit gibt der Ansatz eineraumtheoretische Antwort auf die von Sachs Olsen und Tödtli geforderte Anerkennung einer Vielheit von Raum als einer niemals fixierten Gegebenheit. Denn Lefebvre geht es darum, eine „prozesshafte Denkform zu finden, die erlaubt, sich Objekten anzunähern, diese zu bestimmen, ohne sie in Gänze zu fixieren und sie so gegenüber der Bewegung offen zu halten [...] [und] sich dem Werden und dem Möglichen [zukünftiger Handlungsoptionen] zuzuwenden“ (Lefebvre 1969 [1939]: 32, zit. nach Mullis 2017a: 75), um sich so einem „differenziellen Raum“ (Lefebvre 2006 [1974]: 339) annähern zu können.

\subsection{Lefebvres Theorie von Raum als Produkt sozialer Praxis}

Die zentrale Idee des Aufsatzes „Die Produktion des Raums“ (Lefebvre 2006 [1974]) liegt in der Beschreibung der sozialen Konstitution 
von Raum durch eine Theorie der (räumlichen) Praxis. Lefebvres Praxisbegriff steht dabei in der Tradition marxistischer Theorie. J edoch erweitert er diesen, indem er Raum zwar nach wie vor als Teil der Produktionsmittel denkt, zugleich aber als Produkt einer sozialen Praxis interpretiert (Dünne 2006: 297). Damit liefert Lefebvre einer neomarxistischen Sozialgeographie Anknüpfungspunkte, um die Beziehung von physischem und sozialem Raum neu zu denken. Diese theoretischen Anknüpfungspunkte von Lefebvre, im Folgenden dargestellt nach der Interpretation von Mullis (2017a: 77 ff.), umfassen zwei „nicht deckungsgleich[e], sondern [...] aufeinander verweisend[e] und ineinander verwobene“ Triaden (ebd.: 83).

In der ersten Triade treffen mit Repräsentationen des Raums und Räumen der Repräsentation zwei dialektische Dimensionen sozialer Räumlichkeit aufeinander, vermittelt durch Räumliche Praxis stehen sie sich konflikthaft gegenüber (ebd.: 81; vgl. Abb. 2). Die Repräsentationen

Abb. 2 Soziale Produktion des Raums nach Henri Lefebvre als Ansatz zur Beforschung der Vielheit von Raum (Quelle: eigene Darstellung nach Mullis 2017a)

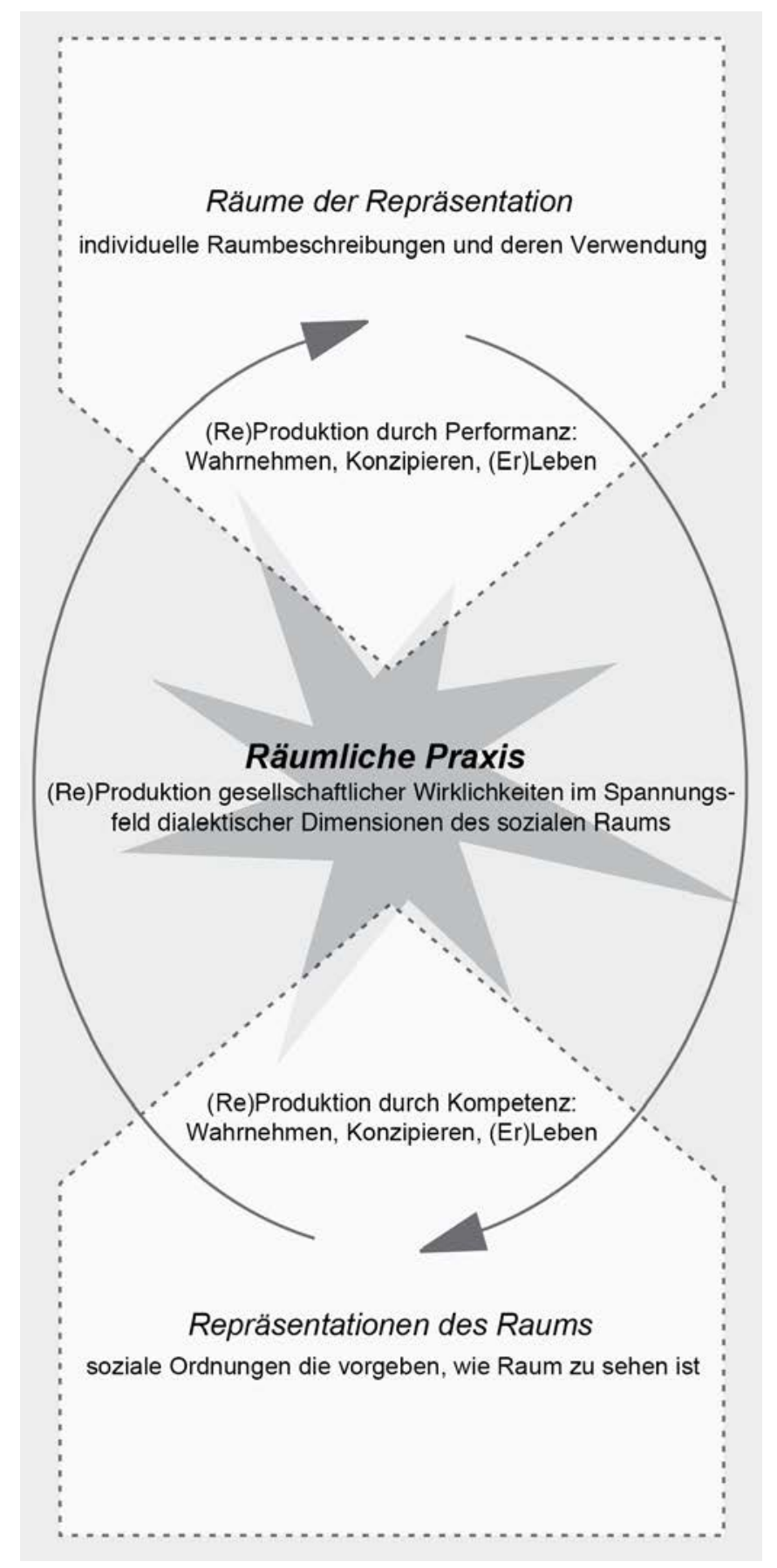


des Raums geben auf mental-konzeptioneller Ebene vor, wie ein Raum zu sehen beziehungsweise zu interpretieren ist. So werden beispielsweise durch die Markierungen öffentlicher und privater Räume oder durch Flächennutzungsplänejene Bedingungen vorgegeben, nach denen sich eine Gesellschaft dem Herrschaftswissen von Raum- und Stadtplaner_innen oder auch Architekt_innen sozial zu fügen hat. Lefebvre spricht in diesem Zusammenhang von sozialen Ordnungen, die von spezifischen Kenntnissen, Zeichen, Codes und konfrontativen Beziehungen durchsetzt sind (Lefebvre 2006 [1974]: 333). Demgegenüber sind die Räume der Repräsentation gekennzeichnet durch sozial produzierte Symbolisierungen; damit verweisen sie auf eine verborgene, nicht offensichtliche Seite des sozialen Lebens. Räume der Repräsentation zeigen sich in individuellen Raumbeschreibungen und deren pragmatischer Verwendungin konkreten (kommunikativen) Kontexten, zum Beispiel in Form von Bildern oder Symbolen „der ,Benutzer, aber auch bestimmter Künstler, [...] Schriftsteller und Philosophen“ (ebd.: 336). Räumliche Praxis wird von Mullis (2017a) hingegen auf einer anderen, grundlegenderen Abstraktionsebene verortet als die beiden konflikt-formierenden Raumdimensionen, da sie ahistorisch und umfassend auf beide Dimensionen zugreift (ebd.: 80). Siereproduziert raum-zeitlich spezifische gesellschaftlich-materielle Wirklichkeiten (Alltag, Stadt, Staat) im „Feld des Möglichen“ (ebd.: 82) und spiegelt dieBedürfnisse und Zwänge ihrer,,sozialen Formationen“ (Lefebvre 2006 [1974]: 333). Dabei zeigt sich, wie sich die Teilhabenden dieser sozialen Formationen - mit ihrer Kompetenz und Performanz (ebd.) - auf den sie umgebenden Raum beziehen. Ergebnisse einer solchen Praxis können Flächennutzungen, Besitzverhältnisse oder materielle Raumausstattungen sein.

Diezweite von Lefebvre entwickelte Triade des wahrgenommenen, konzipierten und gelebten Raums bezieht sich auf räumliche(Re-)Produktionspraxen, wenn diese zu einem Spezifikum der beiden zuvor dargestellten Raumdimensionen werden (Mullis 2017a: 80). Diese Praxen entwickeln sich aus dem Innern der jeweiligen Dimensionen heraus und können innerhalb derselben Dimension in konflikthafter Beziehung zueinander stehen, aber auch eine Spannung der beiden dichotomen Dimensionen bedingen. Der folgendeAbsatzverdeutlicht dieNützlichkeit dieses Modells für die kritische Stadtforschung und zeigt das Potenzial einer Einbindung künstlerischer Strategien in dieses Modell auf.

\subsection{Konflikte in der Räumlichen Praxis und künstlerische Kommentierungen}

Mit Blick auf die westliche mitteleuropäischeStadt verweist Lefebvredarauf, dass historische Entwicklungen das Dominanzverhältnis der beiden dialektischen Dimensionen von sozialem Raum verschoben haben: Während im Mittelalter der soziale Raum auf einen durch religiöse Symboliken geprägten Raum der Repräsentation und auf entsprechend dominante Repräsentationen des Raums reduziert war, vollzog sich im Verlauf der Aufklärungvon der italienischen Renaissance bis zum 19. Jahrhundert ein Wandel des sozialen Raums zu einer widersprüchlichen Vielzahl von Repräsentationen des Raums und Räumen der Repräsentation (Lefebvre 2006 [1974]: 338 f.). Dieser 
Diversifizierungsprozess wurde von großen gesellschaftlichen Konflikten wie der Französischen Revolution begleitet, durch die es zur Abschaffung der absolutistischen Staatsform und zur Durchsetzung von Werten wie bürgerlicher Freiheit und Menschenrechten kam. Für die Argumentation des vorliegenden Textes ist diese Beobachtung wichtig, da es auch in der Gegenwart zu sozialen Spannungen an ebenjenen Berührungspunkten von Repräsentationen des Raums und Räumen der Repräsentation kommt. Dies geschieht beispielsweise, wenn städtische Räume zur Wahrung der Sicherheit systematisch überwacht und dabei von Geboten abweichende individuelleAlltagspraktiken wie die des Sich-Bewegens und des Pausierens im öffentlichen Raum als potenziell verdächtig eingestuft werden oder wenn räumliche Ordnungen im Rahmen von individuellen Raumaneignungen wie Protesten infrage gestellt, unterwandert oder angegriffen werden.

Dieden theoretischen Überlegungen Lefebvres zugrundeliegendeHaltung wurde maßgeblich geprägt von den politischen Ereignissen der 1950er und 1960er Jahre in Frankreich sowie der inhaltlichen Auseinandersetzung mit linken Intellektuellen und Künstler_innen, diezu dieser Zeit in und um Paris lebten. Anne Vogelpohl begründet die gegenwärtig zunehmende Rezeption Lefebvres damit, dass er - geprägt durch die politischen Unruhen von 1968 - gesellschaftlichen Problemen wie Ungleichheit und Entfremdung zu begegnen versuchte. In diesem Sinnerief er dazu auf, diestetig auftretenden Widersprüchezwischen Räumen der Repräsentation und Repräsentationen des Raums sowie daraus resultierende ungleiche Lebensbedingungen in einer (städtischen) Gesellschaft zu analysieren und dadurch zu überwinden (Vogelpohl 2018: 31). Damit erweist sich Lefebvreals unmittelbar anschlussfähigan aktuellestadtgeographische Debatten, in denen „Stadt als ein immer wieder zu stellendes Problem“ (Calbet i Elias et al. 2012: 2) betrachtet wird. Eine Gesellschaftsanalyse, die an sozialen Praktiken ansetzt, kann die (Re-) Produktion der binären Raumdimensionen und die jeweils inhärenten Praxen des Wahrnehmens, Konzipierens, (Er-)Lebens in Bezug auf aktuell vorliegende, raum-zeitlich spezifische gesellschaftliche Wirklichkeiten beziehungsweise urbane Herausforderungen erfassen. Sie kann außerdem ihre konfliktreichen Beziehungen analysieren und somit kommunizieren und nachvollziehbar machen, aus welcher Pluralität von Praxen des Wahrnehmens, Konzipierens und (Er-)Lebens Konflikte entstehen. Dies öffnet Handlungsspielräume, um Differenz sichtbarer und verhandelbarer zu machen und um mit Konflikten leben und umgehen zu lernen.

Im Anschluss an Monika Streule (2014: Abs. 9) kann jedoch eine qualitative Stadtforschung, die sich konzeptionell an Lefebvre orientiert, kaum auf ein künstlerisch orientiertes, transdisziplinär ausgerichtetes Forschen verzichten. Denn die individuell wahrgenommenen und gelebten Räume der Repräsentation entziehen sich sowohl der Alltagssprache als auch den wissenschaftlichen Diskursen. Künstlerische Forschungbietetlaut Streule damit gerade das Potenzial, das Wahrgenommene, Konzipierte und Erlebte im Rahmen künstlerischer Kommentierungen sichtbar zu machen (ebd.: Abs. 10 f.).[6] Vor dem Hintergrund dieses Potenzials künstlerischer Forschung in Konfliktanalysen wird im Folgenden das Konzept der künstlerischen Forschung nach Klein vorgestellt, das einen Ansatz bietet, um zu verstehen und sichtbar zu machen, wie das Verhältnis zwischen 
Repräsentationen des Raums und Räumen der Repräsentation gestaltet und ausgehandelt wird.

In Rückbindung an die Ausführungen zum Begriff der Vielheitvon Raum von Sachs Olsen und Tödtli lässt sich festhalten: Kritische Stadtforschung untersucht eine Vielheit von Raum, wenn sie mit Lefebvre auf eine Betrachtung von Räumlichen Praxen des Vermittelns zwischen den sich konflikthaft gegenüberstehenden und auch intern stetig umkämpften Dimensionen fokussiert (vgl. Abb. 2). Diese Gesellschaftsanalyse wird umfassender, wenn sie auch künstlerische Strategien der Erfassung von Wahrgenommenem, Konzipiertem, Ge- und Erlebtem[7] hinzuzieht. Derim Folgenden vorgestellte Ansatz künstlerischer Forschung ermöglicht es, eine Vielheit von Raum im Sinne einer Vielheit raumbezogener Wissensweisen zwischen den und innerhalb der binären Dimensionen von Raum zu erfassen. Zwar tätigt dieser Ansatz die Verbindung zu Lefebvre nicht selbst, gibt aber vorliegendem Beitrag den gedanklichen Spielraum, ihn analog zur doppelten Triade zu sehen.

\section{Der künstlerische Wahrnehmungsmodus als Ansatz zur Beforschung der Vielheit von Wissen}

Nach der Aufarbeitung der ersten, raumtheoretischen Grundlage ist das Anliegen dieses Kapitels, mit der konzeptionellen Darstellungkünstlerischer Forschungspraxis nach Klein einezweite, erkenntnistheoretischeFundierung vorzunehmen. Beide Ansätze zusammen bilden die Basis, aus der dieser Beitrag eine eigene Forschungsmethodologie entwickelt. Anke Haarmann sieht die Vielzahl an Beiträgen zu künstlerischer Forschung diskursiv und arbeitspraktisch verankert im Kontext von übergeordneten gesellschaftlichen Entwicklungslinien. Aus dieser Perspektive macht sie deutlich, was für eine „monströse Aufgabe“ (Haarmann 2019: 85) es wäre, die Ursprünge künstlerischer Forschung aufzuarbeiten. Für das methodologische Interesse dieses Beitrags ist es zentraler, sich mit der Gemeinsamkeit von künstlerischer und wissenschaftlicher Forschung zu befassen: mit dem Forschen im Sinne einer Praxis (ebd.: 35). Aufbauend auf der wissenschaftlich-raumtheoretischen Basis dieses Beitrags ist es somit relevant, „die Arbeitsweisen und Praktiken der künstlerisch Tätigen [...] ins Zentrum der Aufmerksamkeit [zu rücken], wenn der Begriff des künstlerischen Forschens mit Bedeutung gefüllt werden soll“ (ebd.: 39). Es findet sich im Kontext raumbezogen-künstlerischer Forschungspraxis bereits eine Vielzahl konzeptioneller und forschungspraktischer Ansätze (siehe Endnote 4), die „,art as mode of critical exploration“ (Hawkins 2013: 53) verstehen und in ihren Arbeitsweisen aus dem künstlerischen Bereich schöpfen: zum Beispiel im Sinnevon inventivemethods (Wildner 2015), als transdisziplinäre Praxis von Stadtforschung (Streule 2014) und als ästhetische Praxis, die ein Verhältnis zwischen sinnlichem Erleben und sprachlich-rationalem Begreifen aufspannt (Dickel/Keßler 2019).

Wenn jedoch eine Gesellschaftsanalyse in expliziter Bezugnahme auf Lefebvre von der Betrachtung der sozialen Produktion des Raums ausgeht, so bietet der im Folgenden vorgestellte Ansatz einen Weg, geographischkünstlerische Forschungspraxis begrifflich und verfahrensmäßig zu 
konkretisieren. Denn Kleins Ansatz macht ein theoretisches Angebot, Konflikte anders zu betrachten und mögliche Umgangsweisen zu denken, ohne dabei Kunst und Wissenschaft eine ausschließliche Kompetenz im Sinnlichen oder Analytischen zuzuweisen. Somit stellt der Ansatz die Bedeutung künstlerischer Wahrnehmung für eine systematische und breit angelegteSuchenach Erkenntnis heraus und liefert Anknüpfungspunktefür eine Konturierung der eingangs mit Sachs Olsen und Tödtli als Vielheit von Wissen bezeichneten Pluralität von Wahrnehmungsweisen mittels künstlerischer Forschungspraxen.

\subsection{Zum Begriff des Forschens bei Klein}

Klein verstehtunterkünstlerischerForschungzugleicheineMethode, Strategie oder Praxis des Forschens, welche sich einem spezifisch künstlerischen Modus der Wahrnehmung[8] bedient (Klein 2018: 80 f.). Forschung bezeichnet er als systematische Suche nach neuem Wissen. Er erläutert, dass die Suche ihre Systematik durch stetige Rückgriffe auf (fach-)spezifische theoretische und arbeitspraktische Erfahrung erhält. In Abgrenzung zu Positionen, die Forschen als ausschließlich wissenschaftliche Praxis begreifen, plädiert Klein für einen Forschungsbegriff, der offen für weitere Praxisfelder ist - wie zum Beispiel Industrie, J ournalismus, Kultur oder Künste - und für deren jeweilige Forschungssystematiken. Das Spezifische der jeweiligen Forschungssystematiken sieht er darin, wie Erfahrungen in Praxisfeldern jeweils gesammelt, aufeinander bezogen, transparent gemacht und eingeordnet werden. Erfahrung kann beispielsweise durch Messung gewonnen, sinnlich oder emotional erlebt sowie experimentell erworben werden (ebd.: 82). Eine geeignete Bedingung für die Erweiterung von Wissensbeständen sieht Klein in Forschungsprozessen, dieauf den Erfahrungen und Systematiken mehrerer Praxisfelder aufbauen. Den Künsten weist er hierbei eine Schlüsselrolle zu, da er sie als geschult und geübt darin sieht, Erfahrungen mehrerer Praxisfelder - im Wortlaut „multiplelayers of reality“ (ebd.: 83) - in ihren jeweils spezifischen Zugangsweisen wahrzunehmen und zu reflektieren.

Klein konkretisiert seinen Begriff von reality in diesem Zusammenhang nicht, bezieht künstlerischeWahrnehmungjedoch immerwieder aufDinge: Diese „können Gegenstände sein, Personen, Atmosphären, Vorgänge, aber auch Imaginationen oder Halluzinationen" (Klein 2009: 106). Das Fundament seiner Konzeption künstlerischer Forschung bildet eine Ausführung zur Theaterproduktion, die er als Beispiel nimmt, um vier Betrachtungsweisen beziehungsweise vier Wahrnehmungsmodi zu unterscheiden (ebd.). Daran anknüpfend fokussiert er in einem kurzen Aufsatz mit dem Titel „The Mode is the Method“ einen dieser Modi, den künstlerischen Wahrnehmungsmodus, und fasst ihn als eine erkenntnisorientierte Praxis (Klein 2018). Die daran geknüpften spezifischen Verfahrensweisen umreißt er, arbeitet sie jedoch nicht im Sinne eines forschungsmethodologischen Verfahrens aus. Dies bietet besagten Spielraum, um den Ansatzvon Klein aus der in Kapitel 2 erörterten raumtheoretischen Perspektive zu interpretieren und daraus ein eigenes, raumsensibles Verfahren künstlerischer Forschung zu entwickeln. 


\subsection{Kleins Verortung künstlerischer Forschungspraxis im Konzept der vier Wahrnehmungsmodi}

Dievier - sich in der Praxis einander überlappenden - Modi der Wahrnehmung von Dingen umreißt Klein (2018: 81f.) wie folgt:

- transparent mode/ to do: Art und Weise der unmittelbaren, gegenwärtigen, nicht hinterfragten Wahrnehmung der Existenz eines Dinges.[9]

- aesthetic mode/ to perform: Art und Weise der Wahrnehmung, bei der eine frühere, nicht hinterfragte, subjektive Wahrnehmung von Dingen mit einem spezifischen ästhetischen Erleben verknüpft wird; Wahrnehmung wird hier zu einem ästhetischen Erlebnis, einem Ereignis.

- artisticmode/ to play: Artund WeisederWahmehmungmultipler Realitätsebenen; Wahrnehmung in diesem Modus verknüpft den aesthetic mode und den im Folgenden beschriebenen semiotic mode. Im artistic mode werden vielfältige vorliegende Wahrnehmungsweisen - einerseits ästhetisches Erleben, andererseits etablierte semiotische Repräsentationen - zusammengeführt.

- semiotic mode/ to act: Art und Weise der Wahrnehmung, dieauf bereits spezifisch gedeuteten, gerahmten Repräsentationen wie beispielsweise bildlichen und sprachlichen Zeichen oder Begriffskonzepten basiert, deren Konstruktionscharakter aber im Moment der alltäglichen Wahrnehmung nicht weiter reflektiert wird.

Der dritte, für diesen Beitrag zentrale künstlerische Wahrnehmungsmodus ergibt sich laut Klein - wie bereits angedeutet - aus der Überlappung der angrenzenden Modi (vgl. Abb. 3). ErmachteineVielfalt ästhetischen Erlebens und semiotischen Deutens von Realitäten erfahrbar, setzt sich spielerisch mit deren Übergängen und Grenzen auseinander, entdeckt und hinterfragt deren Unterschiede. Hierbei bestimmt der artistic mode durch den aesthetic mode die Wahrnehmungsweise von Ästhetischem und eröffnet neue Zugänge zu Semiotischem. J edoch entwickelt er im künstlerischen Werk Präsentationen nah am semioticmode, indem er subjektiveästhetischeund semiotischeErfahrungen in ausgewählten Beziehungen und Verkörperungen zeigt und somit spezifisch deutet und rahmt. Die zweifache Ausrichtung am aestheticund am semiotic mode bezeichnet Klein auch als spielerische Funktion des artistic mode. Diese spielerische Funktion beschreibt er, indem er das Kontinuum zwischen Spielen und Nicht-Spielen in vier Schritte unterteilt, die seinen vier Wahrnehmungsmodi entsprechen. Während sich der aesthetic mode in performativer Präsenzund der semiotic modeals aufgeführteRepräsentation zeigen, erscheint der artistic mode als spielerische Präsentation des Vermittelns zwischen aesthetic und semiotic mode (Klein 2018: 80) und wird damit zu einer spielerischen Praxis, die zwischen zwei Bereichen agiert: Einerseits lässt sich der artistic mode in Anlehnung an den aesthetic mode auf einzelne Wahrnehmungsweisen ein. Andererseits betrachtet der artistic mode multipleWahrnehmungsweisen in Anlehnungan den semioticmodeaus einer rahmengebenden Metaperspektive. Zwischen dieser zweifach ausgerichteten Erfahrung baut der artistic mode spielerische Beziehungen zwischen Innenund Außenperspektiven auf (Klein 2009: 122). Dies ergibt insgesamt „drei Erlebens- undVerhaltensweisen“(ebd.: 123), dieden artisticmodeausmachen. 
Abb. 3 Der künstlerische Wahrnehmungsmodus nach Julian Klein als Ansatz zur Beforschung der Vielheit von Wissen (Quelle: eigene Darstellung nach Klein 2018)

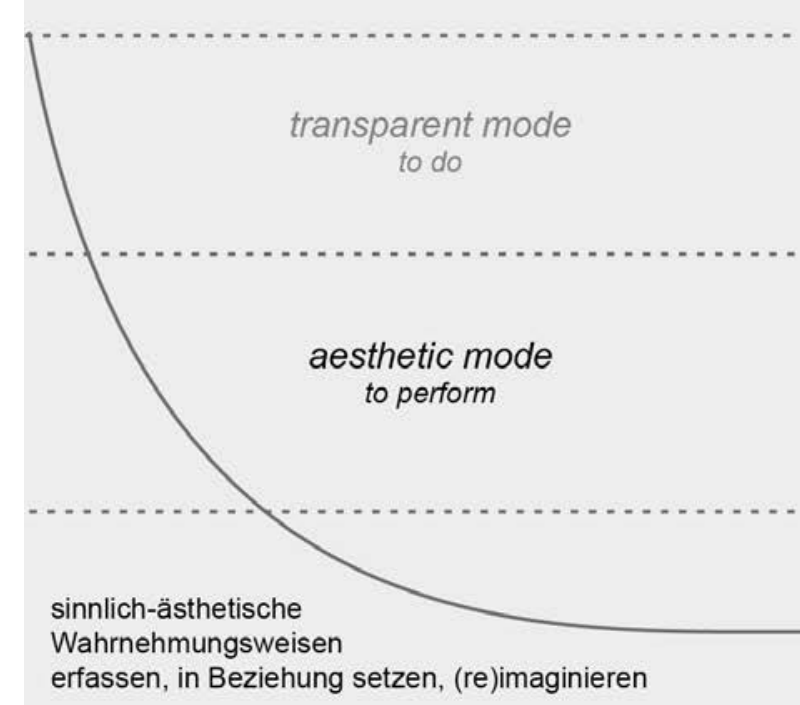

artistic mode

to play

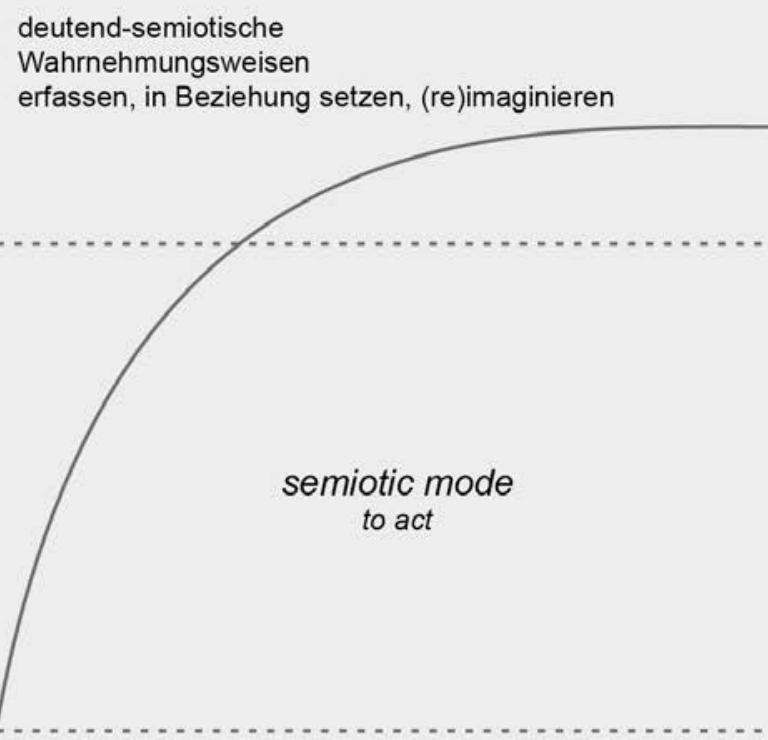

Wildner macht in ihrem Beitrag zu inventive methods, einer Variante künstlerischer Forschung, deutlich, dass es sich beim Spielen mit unterschiedlichen Anordnungen, Settings und Ordnungssystemen um eine vorerst zweckfreie und ergebnisoffene experimentelle Praxis handelt (Wildner 2015: 182). Mitdieser Ergänzungkann das Spielerischedes artisticmode als Zugang verstanden werden, der zwar vorerst zweckfrei, ergebnisoffen und experimentell Möglichkeiten des In-Beziehung-Setzens von Wahrnehmungsweisen und -perspektiven in Erfahrungbringt, jedoch intentional gewählt, systematisch angelegt und auf neue Erkenntnis ausgerichtet ist. Dieser Fokussierung auf Erkenntnisgewinnung entsprechend wird der künstlerische Wahrnehmungsmodus im Folgenden auch als künstlerischer Forschungsmodus bezeichnet. Die erkenntnisbezogene Relevanz des Spiels zwischen Wahrnehmungsweisen und -perspektiven liegt darin, dass der 
artistic mode erscheint als „powerful in being able to bridge the realms of aesthetics and semiotics, because it adds relativity to otherwise nonrelativistic concepts that are still too often recognized as incommensurable" (Klein 2018: 84).

Für die Entwicklung einer systematischen Verfahrensweise künstlerischen Forschens lässt sich Kapitel 3 wie folgt zusammenfassen

(vgl. Abb. 3): Erstens kann aus der Auseinandersetzung mit dem Ansatz von Klein ein Verständnis von Forschung abgeleitet werden, nach dem Akteur_innen in ihrer simultanen Zugehörigkeit zu verschiedenen Gesellschaftsbereichen jeweils spezifisches Wissen produzieren. Künstlerischen Praxen wird dabei die Kapazität zugeschrieben, durch eigene Zugänge zu den Grenzbereichen zwischen diesen spezifischen Weisen der Wissensproduktion neue Erkenntnisfelder zu öffnen. Zweitens kann Kleins artistic mode als systematischer und zugleich spielerischer Zugang zur Erkenntnisgewinnung verstanden werden, der sich aus einer überschneidenden Anwendung zweier angrenzender Wahrnehmungsmodi (aesthetic und semiotic mode) ergibt und für den drei Verfahrensschritte (s. o.) entscheidend sind. Zunächst lässt er sich auf ästhetische und semiotische Wahrnehmungen von Dingen und Vorgängen ein, eignet sie sich an, spürt weitere auf, versammelt sie. Dann betrachtet er diese auf einer Metaebene, setzt sie in temporäre Beziehungen/ Rahmungen und hinterfragt ihre jeweiligen Qualitäten und gegenseitigen Bezüge. Schließlich präsentiert er im künstlerischen Werk mehrere Verkörperungen der erlebten, gesammelten und in Beziehung gesetzten Wahrnehmungen und erschafft oder (re-)imaginiert somit eine eigene semiotische Rahmung konflikthafter, scheinbar inkommensurabler Wahrnehmungsweisen und -perspektiven.

An dieser Stelle lässt sich Kleins erkenntnistheoretischer Ansatz an die Ausführungen zum Begriff der Vielheit von Wissen von Sachs Olsen und Tödtli (2016) rückbinden: Die Überbrückung multipler Wahrnehmungen im künstlerischen Modus durch künstlerische (Re-)Imaginationen schafft eine Kopräsenz von Wahrnehmungsweisen - zum Beispiel zu kontrovers verhandelten städtischen Herausforderungen - , und macht somit eine Koexistenz von scheinbar nicht Vereinbarem möglich (ebd.: 189).

\section{Geographisch-künstlerische Forschungspraxis als Drei-Schritt-Verfahren}

Die konzeptionelle Parallele der beiden Ansätze von Lefebvre und Klein bildet in der Zusammenschau das Fundament für eine Methodologieräumlich-künstlerischer Praxis. Es wurde gezeigt, dass eine Analyse städtischer (Alltags-)Wirklichkeiten mit Lefebvre auf das konflikthafte Verhältnis von Repräsentationen des Raums und Räumen der Repräsentation fokussiert (Kap. 2). Nach diesem Verständnis der sozialen Produktion des Raums kann Kunst als eine engagierte Praxis verstanden werden, die sich mit den vielschichtigen Bezügen von Raumpraxen befasst und dadurch konflikthafte sozialräumliche Verhältnisse (re-)imaginieren und neu artikulieren kann (Kap. 3). Somit lässt sich argumentieren, dass die doppelte Triade der Lefebvre-Interpretation nach Mullis und der artistic mode nach Klein zusammengedacht werden können, indem künstlerische Forschung als eine 
Form Räumlicher Praxis begriffen wird. Diese sucht eine Vielfalt räumlicher Wahrnehmungsweisen - von Sachs Olsen und Tödtli auch als Wissensweisen (Vielheit von Raum/Wissen) bezeichnet - zu erfassen, in Beziehung zu setzen und in $(\mathrm{Re}-$ )Imaginationen zu zeigen. Eine solchePraxis vollzieht eine doppelteNeu-Verknüpfung: Einerseits überbrückt sie die zwei binären Wissensdimensionen des Raumbeschreibens und Raumordnens (vgl. Abb. 2) und andererseits verknüpft sie innerhalb der beiden Dimensionen Praxen des sinnlich-ästhetischen und des deutend-semiotischen Wahrnehmens, Konzipierens, (Er-)Lebens. In der Auseinandersetzung mit urbanen Herausforderungen verbindet räumlich-künstlerische Forschungspraxis somit eine Vielheit konflikthafter Weisen des ästhetischen Raumbeschreibens und semiotischen Raumordnens, indem sie diese Vielheit in drei Schritten herausarbeitet. Dieses Drei-Schritt-Verfahren ist das Ergebnis der in den Kapiteln 1, 2 und 3 aufgebauten Argumentation, das im Folgenden zu einer Methodologie geographisch-künstlerischer Stadtforschung ausgearbeitet und am Beispiel der Projektreihemobileactivators des Architekturkollektivs raumlabor-berlin illustriert wird.

Das Potenzial des hier vorgestellten Verfahrens liegt darin, dass es die Vielschichtigkeit urbaner Problemlagen auf einer Ebeneund in einer Sprache aufgreift, dieüber wissenschaftlich etablierteForschungsmethodologien und auch über Praxen des Integrierens künstlerischer Ausdrucks- und Aufarbeitungsformen hinausgeht, da dieses Verfahren das Wahmehmen, Konzipieren und (Er-)Leben des sinnlich-ästhetischen Raumbeschreibens und des deutend-semiotischen Raumordnens gleichermaßen beforscht und dabei die beiden Dimensionen miteinander und jeweils innerlich neu verknüpft. Dadurch werden - und dies markiert den potenziellen emanzipatorischen Beitrag künstlerischer Forschungspraxen - die Widersprüchlichkeiten Räumlicher Praxen zwischen den alltäglich erfahrenen und scheinbar fixierten Gegebenheiten von Stadt fassbar und lassen sich somit neu verhandeln. Dies hält „den Raum für alle möglichen Zukünfte offen“ (Sachs Olsen/Tödtli 2016: 191), macht das Städtische zu einem „Labor, in dem gesellschaftlich relevantes Wissen erzeugt, erprobt und öffentlich debattiert wird“ (Wildner 2015: 182) und liefert so einen Ausblick auf eine differenzielle städtische Gesellschaft.

Ein Beispiel für eine raumbezogene künstlerische Forschungspraktik in drei Schritten, die für eine stetige und vielschichtige Auseinandersetzung mit Konflikt und Antagonismus im Sinne einer differenziellen Gesellschaft agiert, bieten das Eingangsbeispiel Küchenmonument und die zugehörige Werkgruppe mobile activators des Architekturkollektivs raumlabor-berlin. Im Fokus stehen hierbei „[s]chwierige städtische Orte, [...] die zwischen verschiedenen Systemen, Zeitabschnitten oder Planungsideologien aufgerieben wurden und sich nicht anpassen. Orte, die aufgegeben sind, die übrig bleiben, aber für die Stadtgestalt eine nicht unerhebliche Relevanz haben. "(raumlabor-berlin o.J . b) Solchestädtischen Räumeund Ortenutzt das Kollektiv als soziale und architektonische Experimentierfelder, um zu versuchen, deren Potenziale zu aktivieren (ebd.). Zu den zentralen Arbeitsweisen von raumlabor-berlin gehören urbane Interventionen, durch die städtischeRäumefrei von vorher formulierten Erwartungen verwandelt und zur unvoreingenommenen Wahrnehmung angeboten werden: 
„Wirverschieben programmatische Narrativein städtische Leerräume, installieren neue Atmosphären und schaffen ein Gefühl für neue Potentiale und Freiräume. Durch die Beteiligung der lokalen Akteure in der Zusammenarbeit mit Fachleuten aus allen kreativen Disziplinen werden neue Handlungsfelder entdeckt, getestet und in die Zukunft projiziert."(ebd.)

Beispiele für die Arbeitsweise von raumlabor-berlin bietet die Projektreihe mobile activators: Durch das Installieren pneumatischer Räume wie dem Küchenmonument wird der Stadtraum - zeitlich begrenzt - für alternative kollektive Nutzungen geöffnet und der Möglichkeit Raum gegeben, Qualitäten und Eigenarten zu erkunden und so mit und in architektonischen und sozialen Räumen zu interagieren (vgl. raumlabor-berlin o. J . a). J e nach ortspezifischen Bedingungen und Herausforderungen treten in den Teilprojekten Verfahrensweisen künstlerischer Forschung in beispielhafter Form zutage.

Wiebereits dargestellt, verknüpft der artisticmodedieempirischen Felder des sinnlich-ästhetischen Raumbeschreibens und des deutend-semiotischen Raumordnens auf doppelteWeise durch die Raumpraxen innerhalb der und zwischen den beiden Dimensionen. Der Vollzug dieser Verknüpfung lässt sich in drei Schritten skizzieren (vgl. Abb. 4).[10]

(1) Der erste Schritt, hier als systematische Erfassung und Versammlung vielfältigerWahrnehmungsweisen bezeichnet, fokussiert darauf, die Vielfalt eigener und fremder, sinnlich-ästhetischer und semiotisch-deutenderWahrnehmungsweisen sozialräumlicher Konfliktsituationen zu erfassen, anzueignen und zu versammeln. Diese Erfassung und Versammlung erfordert die Fähigkeit des Sich-Hineinversetzens in Wahrnehmungsweisen aus verschiedensten Kontexten, wie beispielsweise alltägliche ästhetische Erfahrungen und wissenschaftliche Deutungen/Konzepte. Daher sind hier kollaborative, transdisziplinäre Forschungskonstellationen nützlich.

Anhand der Intervention Tempelhof Workshop von raumlabor-berlin wird deutlich, wiedieser erste Schritt in der Praxis aussehen kann: 2016 wurdevor dem alten Empfangsgebäude des früheren Berliner Flughafens Tempelhof für einen Workshop der BerlinerJ ournalistenschule das Küchenmonument aufgebaut. Unter dem Motto „Ein Zelt voller Geschichten“ waren Berliner Schüler_innen sowie in der Unterkunft am ehemaligen Flughafen lebende jugendliche Geflüchtete eingeladen, ihre Medienkompetenz zu stärken und

Abb. 4 Geographischkünstlerische Forschung als DreiSchritt-Verfahren (Quelle: eigene Darstellung)

Drei-Schritt-Verfahren geographisch-künstlerischer Forschung

\begin{tabular}{llll}
\hline Schritt & $(1)$ & $(2)$ & $(3)$ \\
\hline Fokus & $\begin{array}{l}\text { Systematische } \\
\text { Erfassung vielfältiger } \\
\text { Wahrnehmungsweisen }\end{array}$ & $\begin{array}{l}\text { Spielerisches, } \\
\text { ergebnisoffenes \& } \\
\text { experimentell-assoziatives } \\
\text { In-Beziehung-Setzen von (1) }\end{array}$ & $\begin{array}{l}\text { (Re)Imagination } \\
\text { raumbezogener Wissensbestände } \\
\text { und räumlicher Praxen } \\
\text { mittels (2) }\end{array}$ \\
\hline Ziel & $\begin{array}{l}\text { Versammeln vielfältiger } \\
\text { Wahrnehmungsweisen }\end{array}$ & $\begin{array}{l}\text { Verbindungen/Kopräsenzen } \\
\text { entdecken, } \\
\text { deren materielle \& soziale } \\
\text { Voraussetzungen hinterfragen }\end{array}$ & $\begin{array}{l}\text { Präsentation erstellen, } \\
\text { Kopräsenz rahmen, } \\
\text { Koexistenz / Differenz erfahr-, } \\
\text { denk-, kommunizier-, gestaltbar machen }\end{array}$
\end{tabular}


gemeinsam mit dem Team von raumlabor-berlin einen Begegnungsort zu gestalten, mit Gästen aus Politik und Zivilgesellschaft aktuelle Fragen der Flüchtlingsaufnahme zu diskutieren, unter Anleitung von drei geflüchteten Frauen aus Syrien zu kochen und bei alledem einander kennenzulernen, Sprachbarrieren zu überwinden und sich über inhaltliche Fragen auszutauschen (vgl. raumlabor-berlin 2016; Berliner Journalisten-Schule 2016). Die angebotene Raumsituation des Küchenmonuments bildete dabei durch ihre formale und funktionale Veränderlichkeit das Substrat für einen ergebnisoffenen Austausch unterschiedlicher Akteur_innen zu einem Thema, das diese persönlich und ihr Zusammenleben im Stadtraum unmittelbar betrifft.

(2) Der zweite Schritt, hier als spielerisches In-Beziehung-Setzen und Hinterfragen vielfältiger Wahrnehmungen bezeichnet, fokussiert darauf, die im ersten Schritt versammelten heterogenen Wahrnehmungsweisen sozialräumlicher Konfliktsituationen spielerisch, experimentell und ergebnisoffen in Beziehung zu setzen. Dabei werden zunächst bestehende Verbindungen entdeckt oder neue aufgebaut, um anschließend kritisch zu analysieren, welcheimpliziten materiellen und sozialen Voraussetzungen oder Annahmen diese Verbindungen ausmachen.

InZusammenarbeit mit demJ üdischen Museum Frankfurt[11] installierte raumlabor-berlin das Küchenmonumentim Rahmen des zehntägigen Festivals des Museums J udengasse vom 7. bis 17. September 2017 auf dem Frankfurter Willy-Brandt-Platz. Ziel war es, dem zur damaligen Zeit aufgrund von Umbaumaßnahmen geschlossenen Teil des J üdischen Museums am Untermainkai im Rahmen des Festivals eine Präsenz zu geben. Die Blase wurde zum temporären Museum. Besucher_innen waren eingeladen, sich an der materiellen wie inhaltlichen Gestaltung des Pop-up-Museums zu beteiligen: Im Außenraum fanden Workshops zum Bau von Möbeln für die Ausstattung des Stadtraums statt. Im Innenraum konnten dieGästemit den Kurator_innen über deren gestalterische Ideen für das neu zu eröffnende Museum ins Gespräch kommen oder über geplante Ausstellungsinhalte diskutieren und abstimmen (raumlabor-berlin 2017). Abends wurde das Pop-up-Monument dann jeweils mit Lesungen, Filmvorführungen, Konzerten und Diskussionen zum Schauplatzjüdischer Gegenwartskultur bespielt (ebd.). Durch die in und um das Pop-up-Monument angebotenen Einblicke in Planungen und Arbeitsweisen des Jüdischen Museums öffnete sich den Bürger_innen an einem öffentlichen und stark frequentierten Ort ein Raum zum ergebnisoffenen Austausch über persönliche Erlebnisse und Erfahrungen mitjüdischem Leben und jüdischer Kultur in Frankfurt.

(3) Im dritten Schritt, hier als (Re-)Imagination Räumlicher Praxis bezeichnet, werden die Ergebnisse der im zweiten Schritt entwickelten und analysierten Verbindungen im Werk sichtbar. Die (Re)Imaginationen machen eine Vielfalt von Sichtweisen auf Konfliktsituationen erfahrbar, indem sie trotz ihrer scheinbaren Unvereinbarkeit in Kopräsenz gerahmt werden. Diese Präsentationen zielen nicht auf eine konsensorientierte Überwindung von pluralen Sichtweisen ab, sondern bieten Einstiege, die deren Koexistenz erfahr-, denk-, kommunizier- und gestaltbar machen. Künstlerische ( $\mathrm{Re}$-)Imagination beziehungsweise „artistic knowledge“ 
(Klein 2018: 84) trägt in diesem Verständnis dazu bei, neue Formen des Partizipierens zu etablieren, indem über angebotene ( $\mathrm{Re}$-)Imaginationen ein Einstieg in die Auseinandersetzung folgt, der „zu einem Um- und NeuDenken unserer urbanen Geographien und unserem Zusammenleben in den Städten, [...] [führt, sodass] eine räumliche Vielfalt denkbar wird, die schließlich geplant, gebaut und gelebt wird" (Sachs Olsen/Tödtli 2016: 202).

Das Architekturkollektiv raumlabor-berlin dokumentiert seine Projekte - so auch das Küchenmonument - auf seiner Homepage durch Fotostrecken mit kurzen Begleittexten. DieAuseinandersetzungmit dieser Dokumentation offenbart neue Perspektiven und Sichtweisen: So treten in den Fotografien (vgl. z. B. Abb. 1) im Zusammenspiel von gebauter Form (z. B. Hochstraße), pneumatischer Hülleund sozialem Ereignis (z. B. Menschen, diemiteinander kochen, essen und kommunizieren) fluide Situationen in Erscheinung, die momenthaftes Erleben bannen, ungewohnte (Ein-)Blicke eröffnen, Assoziationen anstoßen und schließlich zum Nachdenken anregen. So bietet der Blick auf die fotografische (Re-)Präsentation auch Dritten die Möglichkeit sowohl eines (Nach-)Erlebens der Situation als auch einer Reflexion, etwa über die Atmosphäre von Orten in Abhängigkeit von deren Belebtheit oder über das Verhältnis zwischen autogerechter Stadt und sozialem Miteinander im öffentlichen Raum.

Die dargestellte Abfolge der drei Schritte ist als analytische Stoßrichtung zu verstehen. In der Praxis sind diedrei Schrittejedoch dynamisch miteinander verbunden (Klein 2018: 84). So kann esimkonkreten Fall passieren, dass sie im Prozess des künstlerischen Forschens nicht zwangsläufig nacheinander und aufeinander aufbauend vollzogen werden, sondern sich überlagern (vgl. z. B. Bauer/Nöthen i. E.).

\section{Zusammenfassung und Ausblick}

Mit der Zusammenführung von Lefebvres Theorie zur sozialen Produktion des Raums und Kleins Entwurf künstlerischer Forschung als Praxis des Verknüpfens ästhetischer und semiotischer Wahrnehmungen steht der vorliegende Beitrag zur geographisch-künstlerischen Forschung für einen theoretisch begründeten methodologischen Ansatz, um in einer spezifischen Art und Weise der fragenden Annäherungen an urbane Herausforderungen Stadt aus einer kritischen Perspektive anders zu sehen und zu verstehen. Den zentralen Gedanken des Beitrags bildet das Drei-Schritt-Verfahren, das vielfältigeWahrnehmungen raumbezogener Problemlagen zu erfassen sucht, in Beziehung setzt und zeigt (vgl. Abb. 5). Die Verfahrensschritte wurden in ihrer konzeptionellen Ausrichtung ausgeleuchtet und anhand ausgewählter Projekteaus der Reihemobileactivators des Architekturkollektivsraumlaborberlin aus den J ahren 2006 bis 2017 beispielhaft erläutert.

Hinsichtlich einer Anwendung des Verfahrens ist perspektivisch eine Ausarbeitung konkreter forschungspraktischer Handlungsoptionen und arbeitspraktischer Zugänge zu leisten, die dieästhetischen und semiotischen Modi der Wahrnehmung mit künstlerischen Mitteln spielerisch und kollaborativ zueinander ins Verhältnis setzen. In diesem Sinne haben wir den komplementären Wahrnehmungsmodi entsprechende Handlungsoptionen 
Abb. 5 Drei-

Schritt-Verfahren

geographisch-

künstlerischer

Stadtforschung

als Ansatz zur

Erschließung

der Vielheit

sozialräumlichen

Wissens (Quelle:

eigene Darstellung)

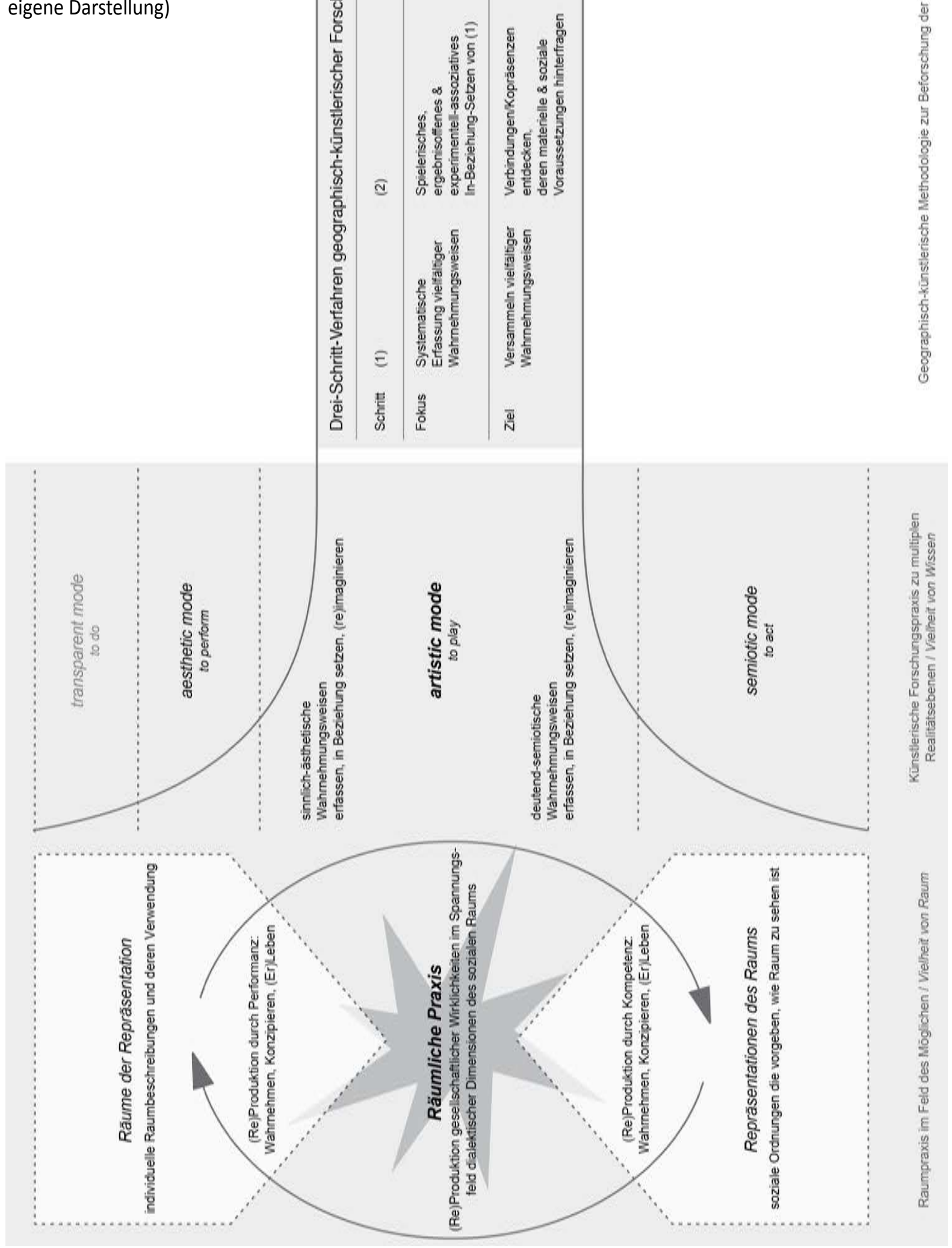


gesammelt und diese den drei Schritten des Verfahrens zugeordnet (vgl. Abb. 6). Diese sollen - als Paarungen angelegt - dazu anregen, ein Spielfeld für künstlerische Forschungspraktiken aufzuspannen. In Anlehnung an die Werkreihe mobile activators liegt bereits ein erster Vorschlag zur (vermittlungs-)praktischen Umsetzung des Verfahrens vor (Nöthen/ Bauer 2021).

Die Ausarbeitung solcher Handlungsoptionen erfordert weitere methodologische Reflexionen zu explorativen, interpretativen und qualitativen Forschungsweisen. Anknüpfungspunkte hierfür finden sich zum Beispiel bei Klein (2009), dem zufolge das Praktizieren des künstlerischen Forschungsmodus eine Haltung individueller und intersubjektiver Rückkopplung erfordert (ebd.: 118 ff.). Bei der individuellen Rückkopplung (mental decoupling) geht es darum, sich zur Überbrückung heterogener Wahrnehmungsweisen von eigenen Perspektiven zu lösen (Klein 2018: 81) ein Aspekt, der auch unter dem Begriff der Selbstreflexivität und der mit Forderung nach einer Situierung der Produktionskontexte von Wissen (vgl. Haraway 1988) verhandelt wird. Die intersubjektive Rückkopplung (meta awareness) macht deutlich, dass individuelle Erfahrung und die InBeziehung-Setzung von Ästhetischem und Semiotischem in kollaborativen Forschungskonstellationen an Konsistenz gewinnen.[12]

Verwendet man das vorgeschlagene Verfahren geographisch-künstlerischer Forschung als methodologischen Ansatz zur Beforschung künstlerisch-wissenschaftlicher Praxen oder greift es in transdisziplinären Forschungskonstellationen auf, so sind neben den genannten Voraussetzungen der Selbstreflexivität und Kollaboration weitere ethische Reflexionen zur methodischen Umsetzung und zum Kontext künstlerischer Wissensproduktion erforderlich. So wird - um beispielhaft nur einen Aspekt herauszugreifen - bezüglich der Wissensproduktionskontexte diskutiert, dass künstlerische Forschungim Rahmen einer zunehmenden Privatisierungvon Wissenschaft und Forschungsförderung teilweise eher einer Erfüllung gesellschaftlicher impact-Kriterien dient, wobei „die der Öffentlichkeit zugewandten performativen Künste zu Modellfällen des [...] impact-Paradigmas instrumentalisiert" (Roms 2013: 215) werden. Dabei werden, beispielsweise im Rahmen von Drittmittelanträgen, künstlerische Ausdrucksformen in der Ergebniskommunikation zur Erfüllung von Anforderungen eines

\section{Abb. 6 Handlungs- optionen geo- graphisch-künstle- rischer Forschung als Drei-Schritt-Verfahren (Quelle: eigene Darstellung)}

\begin{tabular}{|c|c|c|c|}
\hline Schritt & (1) & (2) & (3) \\
\hline Fokus & $\begin{array}{l}\text { Systematische } \\
\text { Erfassung vielfältiger } \\
\text { Wahrnehmungsweisen }\end{array}$ & $\begin{array}{l}\text { Spielerisches, } \\
\text { ergebnisoffenes \& } \\
\text { experimentell-assoziatives } \\
\text { In-Beziehung-Setzen von (1) }\end{array}$ & $\begin{array}{l}\text { (Re)Imagination } \\
\text { raumbezogener Wissensbestände } \\
\text { und räumlicher Praxen } \\
\text { mittels (2) }\end{array}$ \\
\hline Ziel & $\begin{array}{l}\text { Versammeln vielfältiger } \\
\text { Wahrnehmungsweisen }\end{array}$ & $\begin{array}{l}\text { Verbindungen/Kopräsenzen } \\
\text { entdecken, } \\
\text { deren materielle \& soziale } \\
\text { Voraussetzungen hinterfragen }\end{array}$ & $\begin{array}{l}\text { Präsentation erstellen, } \\
\text { Kopräsenz rahmen, } \\
\text { Koexistenz / Differenz erfahr-, } \\
\text { denk-, kommunizier-, gestaltbar machen }\end{array}$ \\
\hline $\begin{array}{l}\text { Handlungs- } \\
\text { optionen }\end{array}$ & $\begin{array}{l}\text { nachdenken } \leftrightarrow \text { wegdenken } \\
\text { registrieren } \leftrightarrow \text { gewahren } \\
\text { verstehen } \leftrightarrow \text { (auf)spüren } \\
\text { erheben } \leftrightarrow \text { sammeln } \\
\ldots \leftrightarrow \ldots\end{array}$ & $\begin{array}{l}\text { experimentieren } \leftrightarrow \text { explorieren } \\
\text { ordnen } \leftrightarrow \text { assoziieren } \\
\text { definieren } \leftrightarrow \text { kombinieren } \\
\text { analysieren } \leftrightarrow \text { konturieren } \\
\ldots \leftrightarrow \ldots\end{array}$ & $\begin{array}{l}\text { formulieren } \leftrightarrow \text { zeigen } \\
\text { publizieren } \leftrightarrow \text { rahmen } \\
\text { schreiben } \leftrightarrow \text { gestalten } \\
\text { argumentieren } \leftrightarrow \text { installieren } \\
\ldots \leftrightarrow \ldots\end{array}$ \\
\hline
\end{tabular}


Wissenstransfers in die breitere Öffentlichkeit integriert, jedoch keine Räume für die Aneignung des künstlerischen Forschungsmodus durch alle Beteiligten geschaffen.

Aktuell wird in der deutschsprachigen kritischen Raumforschung zunehmend Interesse an der Integration wissenschaftlicher und künstlerischer Praktiken sichtbar. Exemplarisch zeigen dies dieJ ahrestagung der Sektion Stadtforschung der Österreichischen Gesellschaft für Soziologie 2021 mit dem Titel „Arts-Based Research in der Stadt- und Raumforschung“, das experimentelle Labor mLab am Geographischen Institut der Universität Bern sowie der von der Arbeitsgruppe „Kritische Geographien globaler Ungleichheiten“ geplante Sammelband Artographies. In diesen Kontexten gilt es, gemeinsam weiter nach Antworten zu suchen auf Fragen wie: Welche Bedingungen ermöglichen es, dieeigenen Wahrnehmungsweisen über eigene Routinen hinaus zu weiten? Wielassen sich kollaborativeProzessegelingend gestalten, sodass verschiedene Formen des Artikulierens eine fruchtbare Beziehungeingehen? WelchePräsentationspraxen erweisen sich als geeignet, um raumbezogene Spannungen im Feld gesellschaftlicher Wirklichkeiten kommunizierbar und perspektivisch gestaltbar zu machen?

Dieser Artikel wurde durch den Open-Access-Publikationsfonds der Universität Leipzig gefördert.

\section{Endnoten}

[1] Wir bedanken uns bei der Redaktion von $\mathrm{s} u \mathrm{~b} \backslash \mathrm{u} \mathrm{r}$ b a $\mathrm{n}$ sowie den anonymen Gutachter_innen für die konstruktiven Rückmeldungen im Begutachtungsprozess, die wesentlich zur Schärfung der Argumentation beigetragen haben. Weiterhin danken wir J ulian Klein für das persönliche Gespräch, Moritz Herrmann, J olina Ulbricht, J ulia Meyer und Anne Köllner für die Durchsicht des Manuskripts, Leon Dreißig für einen wichtigen Literaturhinweis sowie raumlabor-berlin für die Bereitstellung der Fotografie des Küchenmonuments.

[2] Das Kollektiv besteht aus neun Architekt_innen, die sich 1999 zusammengefunden haben, um durch experimentelles Arbeiten an der Schnittstelle zwischen Architektur, Stadtplanung und Kunst einer Stadt von Morgen ein Stück näher zu kommen.

[3] Diese Praktiken beziehen sich auf urbane Prozesse und einhergehende Problemfelder, finden aber nicht zwangsläufig nur in Städten statt. So sind ,urbane Lebensformen in all ihren [...] Facetten dominant geworden und [affektieren] auch Bereiche [...], die auf den ersten Blick nicht urban wirken“ (Mullis 2017b: 362).

[4] Gemeint sind Begriffe wie $\mathrm{a} / \mathrm{r} / \mathrm{t} /$ ography, action research, art as research, art practice as research, artbased research, artful research, artistic research, artled research, artsinformed research, creative inquiry, inventive methods, curating sociology, künstlerische (Feld-)Forschung, Kunst als Forschung, practice as research, practicebased research oder practice-led research. Sie dienen hier der Illustration des Spektrums an unterschiedlichen Hinwendungen zum Themenfeld. Da die Genealogien der Begriffe kaum angemessen zu erfassen sind (Haarmann 2019: 58), wird darauf verzichtet, die Begriffe bestimmten Autor_innen zuzuschreiben. Eine Positionierung zum Forschungsstand künstlerischer Forschung erfolgt in der Einleitung zu Kapitel 3.

[5] Die drei Thesen von Sachs Olsen und Tödtli werden in diesem Text aufgegriffen, indem siedie Strukturierung der folgenden zwei Kapitel entlang der Vielheit von Raum und der Vielheit von Wissen vorgeben. Um den argumentativen Anschluss an Sachs Olsen und Tödtli explizit zu machen, finden sich die zentralen Begriffe ihres Ansatzes in den Titeln dieses und der folgenden beiden Kapitel des Beitrags wieder. 
[6] Streulebezieht sich hier auf die Lefebvre-Interpretation von Schmid (2005), dieim Detail von der Interpretation nach Mullis (2017a) abzugrenzen ist. Nach der Interpretation von Schmid (2005) fallen diebeiden Triaden ineinander, indem Räumeder Repräsentation analog zum gelebten Raum, Repräsentationen des Raums analog zum konzipierten Raum und RäumlichePraxis analog zum wahrgenommenen Raum verstanden werden. Diese Interpretation stützt sich nach Mullis auf Aussagen von Lefebvre, die „alles andere als eindeutig sind“ (ebd.: 80). Die Uneindeutigkeit von Lefebvres Aussagen versteht Mullis als eine frühe, implizite Kritik am marxistischen Begriff des Antagonismus, die deutlicher in der politischen Differenz und der Antagonismus-Konzeption von Laclau und Mouffe formuliert ist (ebd.: 76). Unter Einbezug dieser Perspektive schlägt Mullis vor, den Praxis-Begriff von Lefebvre ,auf einer grundlegenderen Abstraktionsebene als die beiden konkreten, den Konflikt formierenden Positionen zu fassen. Nur so kann [Praxis] auf beide Seiten der binären Konfrontation zugreifen“ (ebd.: 77).

[7] Lefebvre (2006 [1974]) spricht vom gelebten Raum, Mullis (2017a) macht daraus in seiner Abbildung den erlebten Raum. Diese begriffliche Differenz birgt erkenntnistheoretische Implikationen, die für die vorliegende Argumentation jedoch eine untergeordnete Rolle spielen. Daher werden die Begriffe jeweils in Zuordnung zu den Autoren passend und darüber hinaus synonym verwendet.

[8] Während Lefebvre (2006 [1974]) das Wahrnehmen - neben dem Konzipieren und dem (Er-)Leben - als spezifische Reproduktionspraxis von Raum versteht, erklärt Klein (2018) Wahrnehmung zur Prämisse jeglicher Art und Weise des Erkennens von Realitäten.

[9] Dieser Modus ist hier der Vollständigkeit halber aufgeführt. Er ist für die weitere Ausarbeitungjedoch nicht relevant.

[10] FrüheAnsätzeeiner strategischen Fassungkünstlerischer Feldforschungals schrittweisezu vollziehendes Verfahren liegen in den 1970er Jahren bei der Ethnologin und Künstlerin Lili Fischer (vgl. Brenne 2016). Ein kleinschrittigeres Verfahren bieten Stephanie Springgay, Rita L. Irwin und Sylvia Wilson Kind (2005) mit ihrem Ansatz der „enacted living inquiry“ bzw. ,a/r/tography“ (ebd.: 897) an. Auch Wildner (2015) nennt in ihrer Aufarbeitung zu inventive methods Verfahrensweisen, die experimentieren, komponieren und aufführen. Weiterhin benennen Gibson-Graham (2006, zit. nach Baldauf/Hoffner 2016: 328) drei im Grundprinzip ähnlich angelegte Techniken: künstlerische Forschung als epistemologischer Realismus, ontologische Neu-Rahmung und Re-Reading sowie Kreativität der Verschränkung von Referenzrahmen. Diese Ansätze stützen sich jedoch nicht auf die raum- und kunsttheoretischen Spezifizierungen, die vorliegender Beitrag herausarbeitet.

[11] Das Jüdische Museum Frankfurt tritt in der Stadt an zwei Standorten mit spezifischen Schwerpunktsetzungen auf: DasJ üdischeMuseumim Rothschild-Palaisam Untermainkai widmet sich der jüdischen Geschichte und Kultur seit deren gleichberechtigter Eingliederungin diechristliche Mehrheitsgesellschaft. Das Museum J udengassein der Battonnstraßefokussiert auf die Geschichteund Kultur derjüdischen Gemeindein Frankfurt seit der Frühen Neuzeit.

[12] Weitere Argumente für die Positionierung auf einer Metaebene in kollaborativen Forschungskonstellationen liefern geographische Debatten zum epistemological pluralism (Elwood/ Hawkins 2017: 4) und zur künstlerisch-wissenschaftlichen Transdisziplinarität (Streule 2014: Abs. 27). DieAspekte mental decoupling und meta awareness werden in Beiträgen zur künstlerischen Forschung u. a. im Rückgriff auf handlungsleitende Metaphern wie "thinking out of the box“ (Bertram 2018: 82) oder „methodologischer Störsinn“ (Baldauf/Hoffner 2016: 325) besprochen.

\section{Autor_innen}

Lea Bauer ist Humangeographin mit den Schwerpunkten Kritische Kartographie und Politische Geographien.

lea.bauer@uni-leipzig.de

Eva Nöthen ist Geographin und Geographiedidaktikerin mit den Schwerpunkten Visuelle Geographien, Umwelt- und Medienbildung.

noethen@geo.uni-frankfurt.de 


\section{Literatur}

Baldauf, Anette / Hoffner, Ana (2016): Kunst-basierte Forschung und methodischer Störsinn. In: ElkeGaugele/ J ens Kastner (Hg.), Critical Studies. Kultur- und Sozialtheorie im Kunstfeld. Wiesbaden: Springer VS, 325-338.

Bauer, Lea / Nöthen, Eva (i. E.): Kritisches Kartieren als künstlerischer Forschungsmodus. In: Finn Dammann / Boris Michel (Hg.), Handbuch Kritisches Kartieren. Bielefeld: transcript.

Berliner Journalisten-Schule (2016): Medien, Migration und Missverständnisse. https:/ / www.berliner-journalisten-schule.de/ bjs-projekte/ medienprojekt-mitgefl\%C3\%BCchteten/ (letzter Zugriff am 9.6.2021).

Bertram, Ursula (2018): Artistic transfer. Efficiency through unruly thinking. Bielefeld: transcript.

Bertuzzo, Elisa Tullia (2009): Fragmented Dhaka. Analysing everyday life with Henri Lefebvre's theory of production of space. Stuttgart: Steiner.

Brenne, Andreas (2016): Künstlerische Feldforschung. In: Manfred Blohm (Hg.), Kunstpädagogische Stichworte. Hannover: fabrico, 87-90.

Calbet i Elias, Laura / Germes, Mélina / Gribat, Nina / Grotefendt, Nelly / Hutta, Jan / Michel, Boris / Müller, Kristine / Oberländer, Manfred / Praum, Carsten / Roskamm, Nikolai / Schuster, Nina (2012): sublurban. zeitschrift für kritische stadtforschung. Gründungsworkshop, Berlin, 18.-19. Oktober 2012, Positionspapier. https:// zeitschriftsuburban.de/sys/files/docs/Postitionspapier1012.pdf (letzter Zugriff am 9.6.2021).

Dickel, Mirka / Keßler, Lisa (2019): Zwischen sinnlichem Erleben und sprachlich-rationalem Begreifen. Zur Reflexion der ästhetischen Dimension der Forschung in der Geographie. In: Europa Regional 26/1, 49-61.

Dünne, Jörg (2006): Einleitung. Teil IV: Soziale Räume. In: Jörg Dünne / Stephan Günzel (Hg.), Raumtheorie. Grundlagentexte aus Philosophie und Kulturwissenschaften. Frankfurt am Main: Suhrkamp, 289-316.

Elwood, Sarah / Hawkins, Harriet (2017): Intradisciplinarity and visual politics. In: Annals of the American Association of Geographers 107/ 1, 4-13.

Exner, Andreas / Schützenberger, Isabelle (2015): Gemeinschaftsgärten als räumlicher Ausdruck von Organisationsstrukturen. Erkundungen am Beispiel Wien. In: sublurban. zeitschrift für kritische stadtforschung 3/3, 51-74.

Haarmann, Anke (2019): Artistic Research. Eine epistemologische Ästhetik. Bielefeld: transcript.

Haraway, Donna (1988): Situated knowledges. The science question in feminism and the privilege of partial perspective. In: Feminist Studies 14/3, 575-599.

Hawkins, Harriet (2013): Geography and art. An expanding field: Site, the body and practice. In: Progress in Human Geography 37/ 1, 52-71.

Heeg, Susanne (2014): Fragmentierung. In: Julia Lossau / Tim Freytag / Roland Lippuner (Hg.), Schlüsselbegriffe der Kultur- und Sozialgeographie. Stuttgart: Ulmer, 67-80.

Klein, Julian (2009): Zur Dynamik bewegter Körper. Die Grundlage der ästhetischen Relativitätstheorie. In: Julian Klein (Hg.), per.SPICE! Wirklichkeit und Relativität des Ästhetischen. Berlin: Theater der Zeit, 104-134.

Klein, Julian (2018): The mode is the method - or how research can become artistic. In: Daniela J obertová (Hg.), Artistic research: Is there some method? Prague: Academy of Performing Arts, 80-85.

Lefebvre, Henri (1946): Critique de la vie quotidienne. Paris: l'Arche.

Lefebvre, Henri (2006 [1974]): Die Produktion des Raums. In: Jörg Dünne / Stephan Günzel (Hg.), Raumtheorie. Grundlagentexte aus Philosophie und Kulturwissenschaften. Frankfurt am Main: Suhrkamp, 330-340.

Mullis, Daniel (2017a): Krisenproteste in Athen und Frankfurt. Raumproduktionen der Politik zwischen Hegemonie und Moment. Münster: Westfälisches Dampfboot.

Mullis, Daniel (2017b): Henri Lefebvre. Das Recht auf Stadt. In: Frank Eckardt (Hg.), Schlüsselwerke der Stadtforschung. Wiesbaden: Springer VS, 351-366.

Nöthen, Eva / Bauer, Lea (2021): Soziale Grenzfälle in der Stadt. Künstlerisch-forschende Annäherung an urbane Fragmentierung. In: Praxis Geographie 51/ 5, 12-15.

raumlabor-berlin (o. J. a): Mobile Activators. https://raumlabor.net/mobile-activator/ (letzter Zugriff am 9.6.2021).

raumlabor-berlin (o. J . b): Statement. https:// raumlabor.net/ statement/ \#more-62 (letzter Zugriff am 9.6.2021). 
raumlabor-berlin (2016): Tempelhof Workshop. https://raumlabor.net/tempelhofworkshop/ (letzter Zugriff am 9.6.2021).

raumlabor-berlin (2017): Popup Monument. https://raumlabor.net/popup-monument/ (letzter Zugriff am 9.6.2021).

Roms, Heike (2013): Künstlerisch-wissenschaftliche Forschung in den Ruinen der Universität? Performance als wissenschaftliche Veröffentlichungsform. In: Sibylle Peters (Hg.), Das Forschen aller. Artistic Research als Wissensproduktion zwischen Kunst, Wissenschaft und Gesellschaft. Bielefeld: transcript, 205-224.

Sachs Olsen, Cecilie / Tödtli, Sabeth (2016): Re-Imagination des Urbanen. Stadtforschung mit sozial-artistischen Methoden. In: Patrick Oehler / Nicola Thomas / Matthias Drilling (Hg.), Soziale Arbeit in der unternehmerischen Stadt. Wiesbaden: Springer Fachmedien, 187-203.

Schmid, Christian (2005): Stadt, Raum und Gesellschaft. Henri Lefebvre und die Theorie der Produktion des Raumes. Stuttgart: Steiner.

Springgay, Stephanie / Irwin, Rita L. / Wilson Kind, Sylvia (2005): A/r/tography as living inquiry through art and text. In: Qualitative Inquiry 11/6, 897-912.

Streule, Monika (2014): Trend zur Transdisziplinarität. Kritische Einordnung einer ambivalenten Praxis qualitativer Stadtforschung. In: Forum Qualitative Sozialforschung 15/1. https://www.qualitative-research.net/index.php/fqs/article/view/1995 (letzter Zugriff 6.10.2021).

Vogelpohl, Anne (2012): Urbanes Alltagsleben. Zum Paradox von Differenzierung und Homogenisierung in Stadtquartieren. Wiesbaden: Springer VS.

Vogelpohl, Anne (2018): Henri Lefebvre. Die soziale Produktion des Raumes und die urbanisierte Gesellschaft. In: Bernd Belina / Matthias Naumann / Anke Strüver (Hg.), Handbuch kritische Stadtgeographie. Münster: Westfälisches Dampfboot, 30-36.

Wildner, Kathrin (2015): Inventive Methods. KünstlerischeAnsätzein der ethnographischen Stadtforschung. In: EthnoScripts: Zeitschrift für aktuelle ethnologische Studien, 17/ 1, 168-185.

\section{Geographic Artistic Urban Research. A three-step procedure to explore a multiplicity of spatial knowledges}

Art and science use specific approaches to explore urban problems and challenges in their complexity. Despiterecurring mutual references, systematic reflections about connections between artistic approaches in (social) scientific contexts are still rare. However, the epistemological potential of visual and performing arts and their ways of investigating and articulating is increasingly taken up, e.g. in critical urban research. We seea development of research practices, which integrate artistic and scientific approaches in transdisciplinary projects. Drawing on a contribution of Cecilie Sachs Olsen and Sabeth Tödtli on the participatory potential of artistic urban research, this text aims to develop a theoretically grounded methodological approach to geographic-artistic research practice, which allows to see, interpret and co-create different urban spaces from a critical point of view. For this, we bring Henri Lefebvre's theory on the social production of space and Julian Klein's concept of artistic research together. The central focus of this confluence is a three-step process that (1) assembles the diversity of aesthetic and semiotic perceptions of socio-spatial situations, (2) questions and playfully rethinks their many relationships, and (3) presents them in a way that invites participation through the exploration of plural ways of perceiving and seeing the city. Our theoretical considerations are illustrated with examples from selected projects from the architects' collective raumlabor-berlin created between 2006 and 2017. 